\title{
Intervenção Fisioterapêutica no puerpério imediato: O que há de evidências na
}

\section{última década?}

\author{
Physiotherapeutic intervention in the immediate puerperium: What are the evidences in the last \\ decade?
}

Intervención fisioterapéutica en el puerperio inmediato: ¿Cuál es la evidencia en la última década?

Jordânia Abreu Lima de Melo

ORCID: https://orcid.org/0000-0003-3477-4701 Universidade Federal do Rio Grande do Norte, Brasil E-mail: jordaniaabreu@hotmail.com

Luana Brito dos Santos

ORCID: https://orcid.org/0000-0003-2746-7754 Universidade Federal do Rio Grande do Norte, Brasil E-mail: luanabrito02@hotmail.com

Magdalena Muryelle Silva Brilhante

ORCID: https://orcid.org/0000-0002-4246-4351 Universidade Federal do Rio Grande do Norte, Brasil E-mail: mury_brilhante@ hotmail.com

Illia Nadinne Dantas Florentino Lima

ORCID: https://orcid.org/0000-0002-0301-4702 Universidade Federal do Rio Grande do Norte, Brasil E-mail: illialima@yahoo.com.br

Adriana Gomes Magalhães

ORCID: https://orcid.org/0000-0002-0279-5930

Universidade Federal do Rio Grande do Norte, Brasil E-mail: adriana_fsm@yahoo.com.br

\begin{abstract}
Resumo
Objetivo: Analisar a produção científica existente ao longo dos últimos dez anos, referente a atuação fisioterapêutica no puerpério imediato, e o que há de evidências para essa atuação. Métodos: Pesquisa de caráter exploratório do tipo bibliométrica, realizada por meio de uma revisão de literatura nas bases de dados PubMed/Medline, Web of Science, Scopus, PEDro e LILACS, sobre a atuação fisioterapêutica no puerpério imediato publicada na última década. Utilizaram-se os descritores: "postpartum"; "puerperium" e "physiotherapy". Resultados: De 814 publicações, apenas 17 foram analisadas após exclusão de determinadas produções, segundo critérios previamente definidos. Prevaleceram estudos quantitativos, na língua inglesa, realizados no Brasil, publicados nos últimos 5 anos, em periódicos com baixo fator de impacto SJR, que abordaram as temáticas de avaliação e tratamento da diástase dos músculos reto-abdominais (DMRA), seguido pelo uso da eletroestimulação transcutânea (TENS) para alívio da dor no pós-parto imediato e treinamento da musculatura do assoalho pélvico (TMAP). Conclusão: As evidências científicas encontradas apontam como principais intervenções fisioterapêuticas utilizadas no puerpério imediato os exercícios para DMRA, TMAP e TENS porém os estudos apresentam baixo rigor metodológico, e foram publicados em periódicos com baixo fator de impacto. Sugere-se a realização de novos estudos, com maior rigor metodológico, voltados apenas para mulheres no puerpério imediato, para produzir evidências científicas de melhor qualidade.

Palavras-chave: Período pós-parto; Fisioterapia; Modalidades de fisioterapia; Prática Clínica Baseada em Evidências.
\end{abstract}

\begin{abstract}
Objective: To analyze the scientific production of the last ten years, regarding the physiotherapeutic activity in the immediate puerperium and the evidence of this practice. Methods: Exploratory research of the bibliometric type, carried out through a literature review in PubMed/Medline, Web of Science, Scopus, PEDro and LILACS databases, on the physiotherapeutic performance in the immediate puerperium published in the last decade. The following descriptors were used: "postpartum"; "puerperium" and "physiotherapy". Results: Of 814 publications, only 17 were analyzed after exclusion of certain productions according to previously defined criteria. Prevailed the quantitative studies in English, carried out in Brazil, published in the last 5 years in journals with a low SJR impact factor, which addressed the themes of evaluation and treatment of rectus abdominis diastasis (RAD), followed by the use of
\end{abstract}


Research, Society and Development, v. 10, n. 3, e47310312849, 2021

(CC BY 4.0) | ISSN 2525-3409 | DOI: http://dx.doi.org/10.33448/rsd-v10i3.12849

transcutaneos electrostimulation (TENS) for immediate postpartum pain relief and pelvic floor musculature training (PFM). Conclusion: The scientific evidence pointed as the main physiotherapeutic interventions used in the immediate puerperium exercises for RAD, PFM and TENS, but the studies present low methodological rigor and were published in periodicals with low impact factor. It is suggested that new studies with greater methodological rigor be carried out only for women in the immediate puerperium to produce scientific evidence of better quality.

Keywords: Postpartum Period; Physical Therapy Specialty; Physical Therapy Modalities; Evidence-Based Practice.

\section{Resumen}

Objetivo: Analizar la producción científica existente en los últimos diez años, referida a la fisioterapia en el puerperio inmediado, y qué evidencia de esta realización. Métodos: Investigación exploratoria bibliométrica, realizada a través de una revisión de la literatura en las bases de datos PubMed / Medline, Web of Science, Scopus, PEDro y LILACS, sobre el desempeño fisioterapéutico en el puerperio inmediato publicado en la última década. Se utilizaron los siguientes descriptores: "postpartum"; "puerperium" y "physiotherapy". Resultados: de 814 publicaciones, solo 17 fueron analizadas tras excluir determinadas producciones, según criterios previamente definidos. Estudios cuantitativos, en inglés, realizados en Brasil, publicados en los últimos 5 años, en revistas con factor de impacto bajo SJR, que abordaron los temas de evaluación y tratamiento de la diástasis de los músculos rectos del abdomen (DMRA), seguidos del uso de Electroestimulación transcutánea (TENS) para el alivio del dolor en el posparto inmediato y entrenamiento de los músculos del suelo pélvico (TMAP). Conclusión: La evidencia científica encontrada señala que los ejercicios DMRA, TMAP y TENS son las principales intervenciones de fisioterapia utilizadas en el puerperio inmediato, pero los estudios tienen bajo rigor metodológico y han sido publicados en revistas con bajo factor de impacto. Se sugiere que se realicen más estudios, con mayor rigor metodológico, dirigidos únicamente a mujeres en el puerperio inmediato, para producir evidencia científica de mejor calidad.

Palabras clave: Período posparto; Fisioterapia; Modalidades de fisioterapia; Práctica clínica basada en evidencias.

\section{Introdução}

O puerpério é o período que se inicia após o parto, sendo dividido em imediato que dura de 1 a 10 dias, tardio de 11 a 45 dias ou remoto após 45 dias (Andrade, Santos, Maia \& Mello, 2015). O pós-parto imediato é um período marcado por modificações biológicas e psicossociais que ocorrem no organismo da mulher pelo retorno do seu corpo ao estado anterior a gestação, bem como na sua adaptação ao papel materno (Mesquita, et al., 2019).

As alterações físicas, emocionais e sociais (Haran, Van Driel, Mitchell \& Brodribb, 2014), que embora sejam em sua maioria fisiológicas, podem favorecer o surgimento de complicações (Lavand'homme, 2013), que perpassam as mudanças hemodinâmicas (Gardenghi, Dezotti, Dalio, Joviliano \& Piccinato, 2017), o aparecimento da diástase dos músculos retoabdominais (Rett, et al., 2014), constipação e diminuição do peristaltismo intestinal decorrentes dos ajustes hormonais (Rett, Bernardes, Santos, Oliveira \& Andrade, 2008), alterações na função pulmonar atribuíveis aos hormônios e ao impedimento mecânico causado pelo feto em crescimento (Siddiqui, Tauheed, Ahmad \& Mohsin, 2014; Lemos, Souza, Andrade, Figueiro \& Filho, 2011), alterações posturais e aparecimento de complicações nas mamas, proveniente da posição incorreta de amamentar (Goyal, Banginwar, Ziyo \& Toweir, 2011; Witt, Bolman, Kredit \& Vanic, 2016), assim como alterações no músculos do assoalho pélvico que podem contribuir para o surgimento de algumas disfunções, como a incontinência urinária (Pizzoferrato, et al., 2016).

Para garantir uma assistência adequada, o Ministério da Saúde vem formulando políticas que garantam melhoria na assistência desde o pré-natal até o puerpério (Brasil, 2011), e dentre as profissões que podem contribuir para amenizar as alterações persistentes do período gestacional até o pós-parto, é a fisioterapia (Unsgaard-Tøndel, Vasseljen, Woodhouse \& Mørkved, 2016), que surge como tratamento eficaz para reduzir potenciais fatores que podem comprometer a mulher nessa fase, iniciando e implementando intervenções desde o puerpério imediato (Santana, Gallo, Marcolin, Ferreira \& Quintana, 2011).

A literatura científica ainda é escassa no que diz respeito aos cuidados oferecidos pelo fisioterapeuta no puerpério imediato (Vanderkruik, Tunçalp, Chou \& Say, 2013), mas já se sabe, que a atuação fisioterapêutica trata alterações 
musculoesqueléticas (Ashrafinia, et al., 2015), disfunções uroginecológicas (Wu, McInnes \& Leong, 2018), dores (Teymuri, Hosseinifar \& Sirousi, 2018; Saleh, Botla \& Elbehary, 2018), corrigindo posturas (Crockett, et al., 2018) e promovendo exercícios físicos (Theodorsen, Strand \& Bø, 2018) e cardiorrespiratórios (Pinto, et al., 2015), orientando a amamentação e as atividades de vida diária (Evenson, Owe, Rousham \& Brown, 2014; Falcão, et al., 2015; Burti, Cruz Silva \& Moreira, 2016). Porém, apesar das inúmeras intervenções que a fisioterapia pode aplicar no intuito de tratar e prevenir complicações ao longo do puerpério, se faz necessário entender em que ponto se encontra a produção científica no que tange a atuação fisioterapêutica no puerpério imediato, neste caso a pesquisa bibliométrica pode ser uma ferramenta estratégica para apontar tais resultados.

Diante disso, este artigo apresenta um estudo bibliométrico com a finalidade de analisar a produção científica existente ao longo dos últimos 10 anos, referente a atuação fisioterapêutica no puerpério imediato, buscando artigos indexados, em bases de dados nacionais e internacionais, para construir um panorama acerca dos avanços na produção científica que ocorreram ao longo desse tempo.

\section{Metodologia}

Esta é uma pesquisa de caráter exploratório, na qual utilizou-se os conceitos da pesquisa bibliométrica para o planejamento, execução da coleta e análise dos dados coletados.

Sendo a pesquisa bibliométrica uma estratégia matemática para análise, identificação e descrição de padrões na produção científica, diante de determinado tema, permitindo apontar como resultado as características da produção científica existente (Araújo, 2006), sintetiza as informações, e gera um panorama dos elementos textuais presentes na literatura (Martinez-Pulgarin, Acevedo-Mendoza, Cardona-Ospina, Rodríguez-Morales \& Paniz-Mondolfi, 2016), favorecendo a origem de novas informações, auxiliando na tomada de decisão e possibilitando a identificação de tendências em cada um dos setores no qual se busca a informação (Machado, 2007).

Como questão norteadora do estudo elegeu-se a seguinte indagação: "Intervenção fisioterapêutica no puerpério imediato, o que há de evidências?".

Para isso, se fez necessário o seguimento das seguintes etapas de pesquisa:

1. Revisão de literatura sobre a produção científica envolvendo a fisioterapia e o puerpério;

2. Análise bibliométrica dos artigos indexados, caracterizando-os em tipologias;

3. Registro das informações em um protocolo padronizado;

4. Descrição dos dados obtidos;

5. Análise dos resultados encontrados.

Inicialmente, a busca de dados foi realizada por meio de uma revisão de literatura junto as bases de dados PubMed/Medline, Web of Science, Scopus, PEDro e LILACS, tendo como direcionamento a produção científica nacional e internacional, sobre a atuação fisioterapêutica no puerpério imediato ao longo dos últimos 10 anos. Como estratégia de busca em cada uma das bases de dados, foram utilizados os descritores Decs/Mesh: "postpartum"; "puerperium" $e$ "physiotherapy", sendo combinados com o operador boleano “AND”.

Finalizada a etapa da revisão bibliográfica, seguiu-se com a análise do material encontrado. Todas as publicações foram avaliadas pelos títulos, resumos e pela íntegra, sendo excluídas aquelas em que a temática não correspondia ao objetivo da pesquisa, foram incluídas publicações de todos os países, sem restrição linguística. 
Research, Society and Development, v. 10, n. 3, e47310312849, 2021

(CC BY 4.0) | ISSN 2525-3409 | DOI: http://dx.doi.org/10.33448/rsd-v10i3.12849

\section{Resultados}

Foi analisado neste estudo bibliométrico a produção científica sobre a atuação da fisioterapia no puerpério imediato durante o período de 2009 a 2019, produzida nacionalmente e internacionalmente.

A partir da análise do Quadro 1 é possível verificar as produções científicas que foram encontradas em cada base de dados, distribuídas de acordo com o número de artigos encontrados, e a porcentagem desses diante de todos os artigos incluídos: 15 (51,7\%) artigos encontrados na PubMed/Medline, 8 (27,6\%) na Scopus, 3 (10,3\%) na Web of Science, 2 (7\%) na LILACS e $1(3,4 \%)$ na PEDro, totalizando um número de 29 artigos incluídos e após exclusão das duplicatas (12) restaram 17 artigos para análise, tendo a PubMed/Medline se destacado com o maior número de artigos encontrados. Os dados analisados propiciaram a identificação dos autores que publicam nessa área, a frequência de publicação ao longo desses 10 anos, os periódicos, a língua, o país de origem, entre outras informações, que contribuíram na caracterização acerca da produção científica existente a nível nacional e internacional. 


\section{Quadro 1. Estratégias de busca e resultados encontrados nas bases de dados}

\begin{tabular}{|c|c|c|}
\hline Base de Dados & Expressão de busca & Número de artigos encontrados \\
\hline \multirow[b]{2}{*}{ PUBMED/Medline } & Postpartum AND physiotherapy & $\begin{array}{l}\text { Geral: } 613 \\
\text { Últimos } 10 \text { anos: } 345 \\
\text { Excluídos após leitura do título: } 240 \\
\text { Excluídos após leitura do resumo: } 64 \\
\text { Excluído após leitura completa: } 26 \\
\text { Sem acesso ao artigo completo: } 3 \\
\text { Incluídos: } 12\end{array}$ \\
\hline & Puerperium AND physiotherapy & $\begin{array}{l}\text { Geral: } 385 \\
\text { Últimos } 10 \text { anos: } 206 \\
\text { Excluídos após leitura do título: } 102 \\
\text { Excluídos após leitura do resumo: } 77 \\
\text { Excluído após leitura completa: } 20 \\
\text { Sem acesso ao artigo completo: } 4 \\
\text { Incluídos: } 3\end{array}$ \\
\hline \multirow[t]{2}{*}{ Web of Science } & Postpartum AND physiotherapy & $\begin{array}{l}\text { Geral: } 60 \\
\text { Últimos } 10 \text { anos: } 45 \\
\text { Excluídos após leitura do título: } 16 \\
\text { Excluídos após leitura do resumo: } 14 \\
\text { Excluído após leitura completa: } 9 \\
\text { Sem acesso ao artigo completo: } 3 \\
\text { Incluídos: } 3\end{array}$ \\
\hline & Puerperium AND physiotherapy & $\begin{array}{l}\text { Geral: } 1 \\
\text { Últimos } 10 \text { anos: } 0 \\
\text { Não foi incluído }\end{array}$ \\
\hline \multirow{2}{*}{ Scopus } & Postpartum AND physiotherapy & $\begin{array}{l}\text { Geral: } 176 \\
\text { Últimos } 10 \text { anos: } 119 \\
\text { Excluídos após leitura do título: } 78 \\
\text { Excluídos após leitura do resumo: } 15 \\
\text { Excluído após leitura completa: } 16 \\
\text { Sem acesso ao artigo completo:4 } \\
\text { Incluídos: } 6\end{array}$ \\
\hline & Puerperium AND physiotherapy & $\begin{array}{l}\text { Geral: } 138 \\
\text { Últimos } 10 \text { anos: } 78 \\
\text { Excluídos após leitura do título: } 51 \\
\text { Excluídos após leitura do resumo: } 8 \\
\text { Excluído após leitura completa: } 14 \\
\text { Sem acesso ao artigo completo: } 3 \\
\text { Incluídos: } 2\end{array}$ \\
\hline \multirow[t]{2}{*}{ PEDro } & Postpartum AND physiotherapy & $\begin{array}{l}\text { Geral: } 15 \\
\text { Últimos } 10 \text { anos: } 13 \\
\text { Excluídos após leitura do título: } 2 \\
\text { Excluídos após leitura do resumo: } 6 \\
\text { Excluído após leitura completa: } 2 \\
\text { Sem acesso ao artigo completo: } 2 \\
\text { Incluídos: } 1\end{array}$ \\
\hline & Puerperium AND physiotherapy & Geral: 0 \\
\hline \multirow{2}{*}{ LILACS } & Postpartum AND physiotherapy & $\begin{array}{l}\text { Geral: } 12 \\
\text { Últimos } 10 \text { anos: } 4 \\
\text { Excluídos após leitura do título: } 1 \\
\text { Excluído após leitura completa: } 2 \\
\text { Incluídos: } 1\end{array}$ \\
\hline & Puerperium AND physiotherapy & $\begin{array}{l}\text { Geral: } 12 \\
\text { Últimos } 10 \text { anos: } 4 \\
\text { Excluídos após leitura do título: } 2 \\
\text { Excluído após leitura completa: } 1 \\
\text { Incluídos: } 1\end{array}$ \\
\hline
\end{tabular}

Fonte: Elaborado pelas autoras. 
Research, Society and Development, v. 10, n. 3, e47310312849, 2021

(CC BY 4.0) | ISSN 2525-3409 | DOI: http://dx.doi.org/10.33448/rsd-v10i3.12849

A organização e seleção das etapas da pesquisa encontram-se na Figura 1.

Figura 1 - Fluxograma dos estudos incluídos na revisão

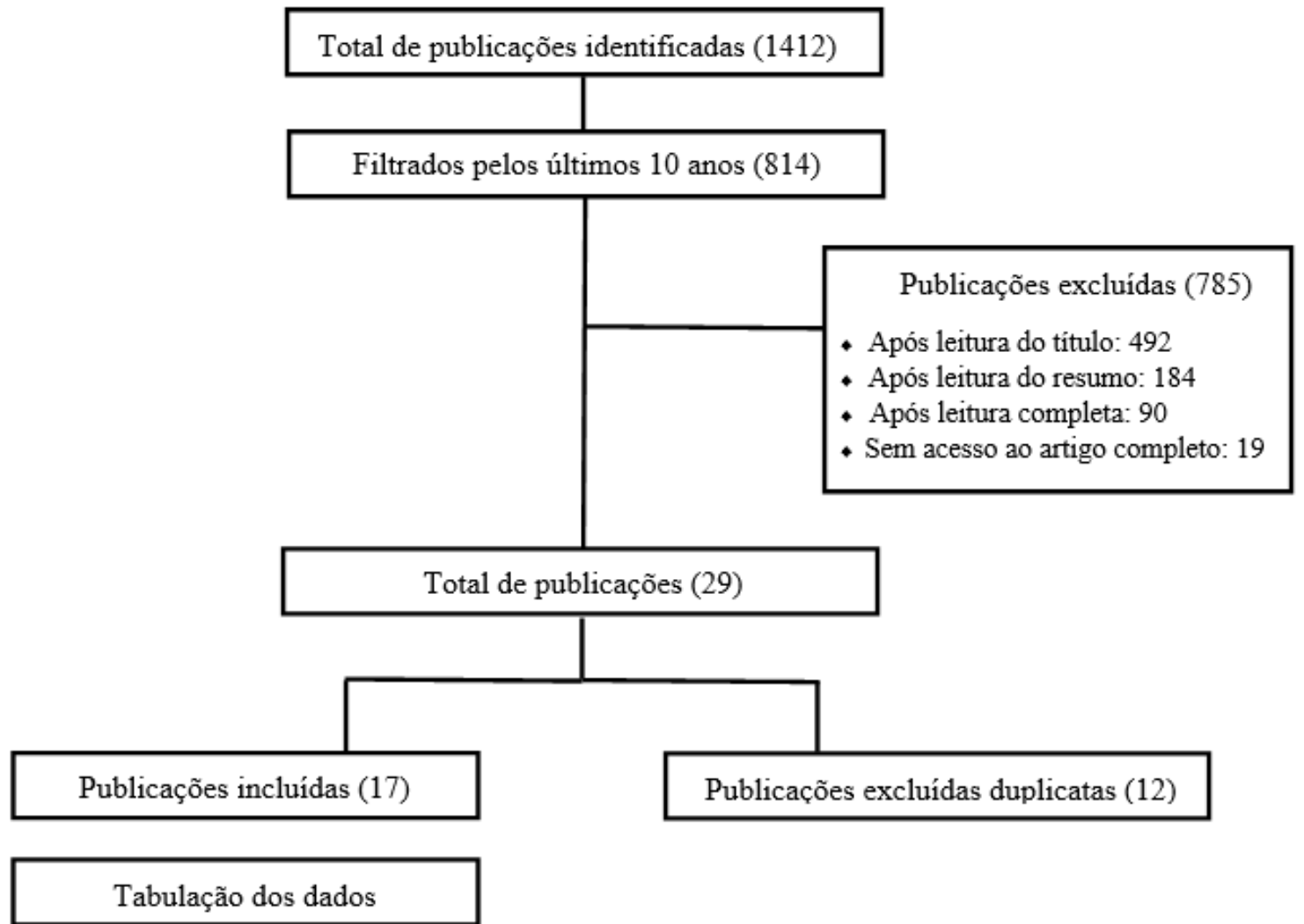

Análise e interpretação dos dados

Redação do texto final

Fonte: Elaborado pelas autoras, a partir das bases de dados.

O gráfico 1 apresenta os resultados da distribuição do número de publicações por temáticas abordadas com maior frequência: exercícios para diminuir diástase dos músculos reto-abdominais (DMRA) (4), estimulação elétrica nervosa transcutânea (TENS) (3), exercícios para os músculos do assoalho pélvico (MAPS) (3), pilates (2), exercício e atividade física (2), exercícios para diminuir DMRA associada a contração dos MAPS (1), crioterapia (1), exercícios de Kegel (EK) e estimulação elétrica intravaginal de baixa frequência e voltagem (DES) (1). 
Research, Society and Development, v. 10, n. 3, e47310312849, 2021

(CC BY 4.0) | ISSN 2525-3409 | DOI: http://dx.doi.org/10.33448/rsd-v10i3.12849

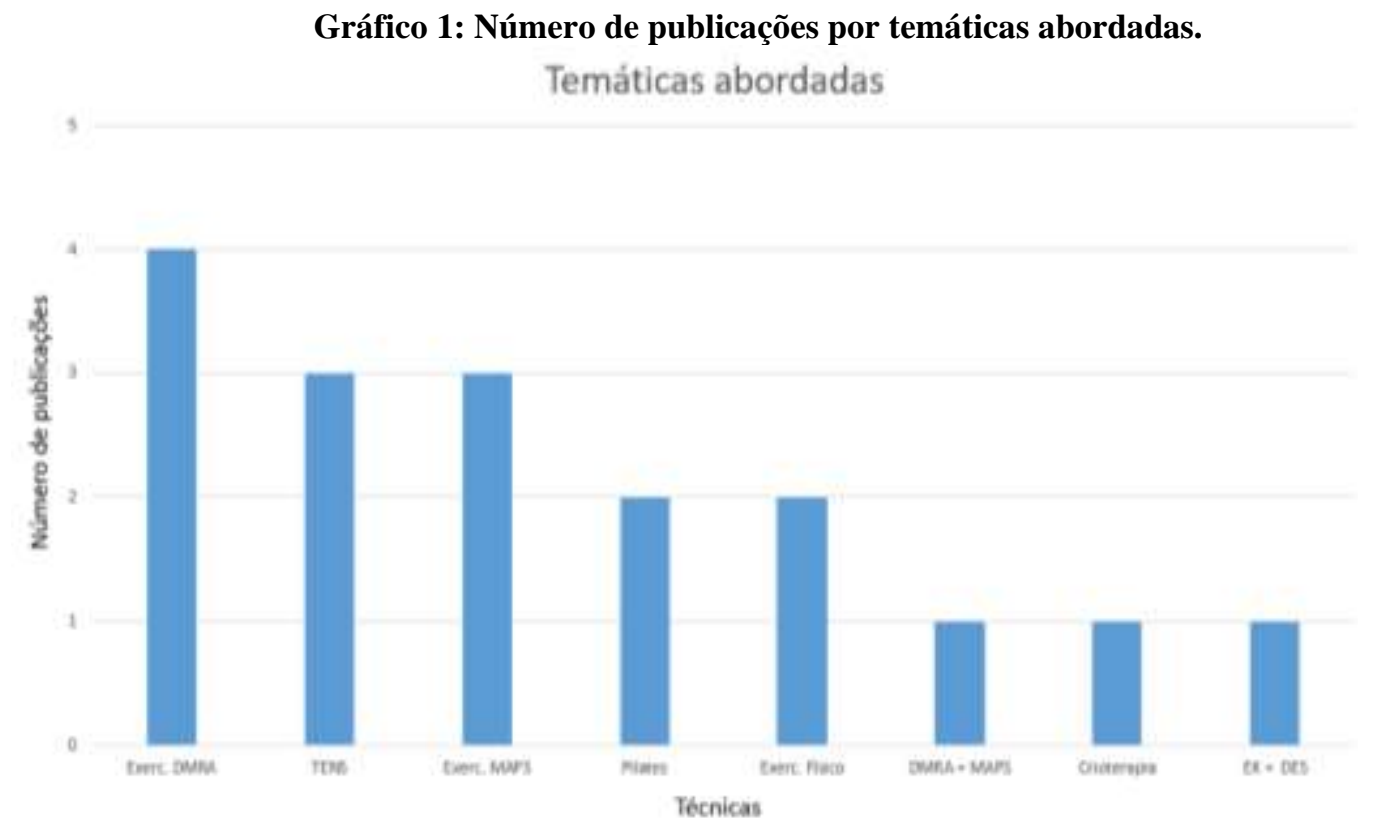

Legenda: Exerc. DMRA: exercícios para diminuir diástase dos músculos reto-abdominais; TENS: estimulação elétrica nervosa transcutânea; Exer. MAPS: exercícios para os músculos do assoalho pélvico; DMRA + MAPS: exercícios combinados para diminuir DMRA associada a contração dos músculos do assoalho pélvico; EK + DES: exercícios combinados: exercícios de Kegel e estimulação elétrica intravaginal de baixa frequência e voltagem.

Fonte: Elaborado pelas autoras, a partir das bases de dados.

Pode-se perceber uma intensificação na produção dos estudos em avaliação e tratamento da DMRA, seguido pelo uso da eletroestimulação transcutânea para alívio da dor no pós-parto imediato e treinamento da musculatura do assoalho pélvico.

O maior número de publicações, concentraram-se nos últimos 5 anos, tendo em vista que a maior quantidade de artigos publicados foram no ano de 2018 (6), seguido por 2017 (3), 2015 (3) e 2014 (2). Os demais artigos foram publicados no ano 2016 (1), 2013 (1) e 2009 (1), demonstrando dessa forma que as pesquisas nessa área vêm crescendo gradualmente, porém muitas lacunas ainda permanecem em aberto, o que reforça a necessidade de mais estudos (Quadro 2).

No quadro 2 é possível observar informações quanto ao país de origem, onde foi encontrado um maior número de artigos produzidos no Brasil (4), seguidos da Polônia (3) e Irã (2). A língua inglesa foi predominante estando presente em 12 dos 17 artigos, e os periódicos que possuem mais publicações relacionadas ao tema de busca são: Current Women's Health Reviews (2) e Physiotherapy (2), ambos apresentam fator de impacto SJR de 0,127 e 1,085, respectivamente.

No que concerne à natureza do estudo, a grande maioria foram estudos quantitativos, como já é bastante evidenciado na literatura quando se refere a pesquisas da área da saúde. Sendo o tipo de estudo mais encontrado, os ensaios clínicos randomizados controlados (8), seguidos das revisões, englobando as sistemáticas, as de literatura e as narrativas, que juntas apresentaram um total de 7 artigos (Quadro 2).

Muitas das revisões encontradas (5) buscaram na literatura estudos que avaliavam e tratavam a DMRA desde a gestação, até o pós-parto tardio, e foram incluídas pois o período do pós-parto imediato é perpassado ao longo do acompanhamento realizado também durante essa fase, porém elas abordam a assistência fisioterapêutica de forma longitudinal, sendo ofertada nas fases de pré-parto e pós-parto imediato e tardio. A abordagem desta pesquisa por sua vez, busca quantificar a produção científica acerca dessa temática, com enfoque na assistência prestada pela fisioterapia durante o puerpério imediato, visto que, não foi encontrada na literatura essa estratégia de medida sobre esse tema, destacando-se assim a necessidade de analisar a produção científica existente ao longo dos últimos 10 anos, por meio de um estudo bibliométrico. 
Quadro 2. Descrição dos artigos

\begin{tabular}{|c|c|c|c|c|c|c|c|c|}
\hline Primeiro Autor & Ano & Natureza do Estudo & Tipo de Estudo & $\begin{array}{l}\text { Periódico/Fator de } \\
\text { impacto SJR } 2018\end{array}$ & País & Língua & Intervenção & Tema \\
\hline 1. Patricia Mota & 2015 & $\begin{array}{c}\text { Descritiva documental - } \\
\text { Quantitativo }\end{array}$ & Revisão de literatura & $\begin{array}{l}\text { Current Women's Health } \\
\text { Reviews/0,127 }\end{array}$ & Portugal & Inglesa & Sem intervenção & $\begin{array}{c}\text { Fatores de risco, implicações } \\
\text { funcionais e o efeito do exercício } \\
\text { na DMRA durante a gestação e } \\
\text { após o parto }\end{array}$ \\
\hline $\begin{array}{l}\text { 2. Laura Anne } \\
\text { Werner }\end{array}$ & 2018 & $\begin{array}{l}\text { Descritiva documental- } \\
\text { Quantitativo }\end{array}$ & Revisão sistemática & $\begin{array}{l}\text { Current Women's Health } \\
\text { Reviews/0,127 }\end{array}$ & EUA & Inglesa & Sem intervenção & $\begin{array}{l}\text { Exercícios pra DMRA associado a } \\
\text { contração dos MAP durante a } \\
\text { gestação e após o parto }\end{array}$ \\
\hline 3. Siv Mørkved & 2018 & $\begin{array}{c}\text { Descritiva documental - } \\
\text { Quantitativo }\end{array}$ & Revisão sistemática & $\begin{array}{l}\text { British Journal of Sports } \\
\text { Medicine/4,141 }\end{array}$ & Noruega & Inglesa & Sem intervenção & $\begin{array}{c}\text { Prevenção e tratamento da } \\
\text { incontinência urinária com o } \\
\text { treinamento dos MAP durante a } \\
\text { gestação e após o parto }\end{array}$ \\
\hline $\begin{array}{l}\text { 4. Agnieszka } \\
\text { Opala-Berdzik }\end{array}$ & 2009 & $\begin{array}{c}\text { Descritiva documental - } \\
\text { Quantitativo }\end{array}$ & Revisão de literatura & Physiotherapy/1,085 & Polônia & Polonesa & $\begin{array}{c}\text { Exercício abdominal concêntrico, durante } \\
\text { a gestação } \\
\text { 2x/dia até } 10 \text { rep. } \\
\text { E a partir do } 3^{\circ} \text { dia pós-parto } 5 \times 10 \\
\text { rep./dia. }\end{array}$ & $\begin{array}{l}\text { Proposta de procedimentos } \\
\text { fisioterapêuticos baseados na } \\
\text { literatura, para tratamento na } \\
\text { DMRA em gestantes e puérperas }\end{array}$ \\
\hline $\begin{array}{l}\text { 5. Patrycja } \\
\text { Żaneta } \\
\text { Bobowik }\end{array}$ & 2018 & $\begin{array}{c}\text { Intervenção Clínica - } \\
\text { Quantitativo }\end{array}$ & $\begin{array}{l}\text { Ensaio Clínico } \\
\text { Randomizado e } \\
\text { Controlado }\end{array}$ & $\begin{array}{c}\text { Posteppy } \\
\text { Rehabilitacji/0,102 }\end{array}$ & Polônia & Polonesa & $\begin{array}{l}\text { Mulheres entre 0-3 dias após o parto, com } \\
\text { DMRA }>2 \mathrm{~cm}, 40 \text { mulheres foram } \\
\text { randomizadas em um grupo que realizou } \\
\text { um programa fisioterapêutico dividido em } \\
3 \text { partes, durante } 6 \text { semanas. E as outras } \\
20 \text { não realizaram intervenção. }\end{array}$ & $\begin{array}{c}\text { Programa fisioterapêutico voltado } \\
\text { para redução de DRAM em } \\
\text { mulheres no pós-parto imediato. }\end{array}$ \\
\hline $\begin{array}{l}\text { 6. Elaine Maria } \\
\text { Souza Alves }\end{array}$ & 2015 & $\begin{array}{c}\text { Intervenção Clínica - } \\
\text { Quantitativo }\end{array}$ & $\begin{array}{l}\text { Ensaio Clínico } \\
\text { Randomizado e } \\
\text { Controlado }\end{array}$ & Revista Dor/0,1852 & Brasil & Portuguesa & $\begin{array}{c}\text { Puérperas no pós-operatório de cesariana } \\
\text { que tivesse de } 8 \text { até } 24 \text { h pós-parto foram } \\
\text { submetidas a eletroestimulação nervosa } \\
\text { transcutânea durante } \\
30 \text { minutos, com frequência de } 100 \mathrm{~Hz} \text { e } \\
\text { largura de pulso } 100 \mu \mathrm{s} \text {. }\end{array}$ & $\begin{array}{l}\text { Eletroestimulação nervosa } \\
\text { transcutânea para analgesia pós- } \\
\text { parto imediato de cesariana. }\end{array}$ \\
\hline 7. Erika Mihelj & 2018 & $\begin{array}{c}\text { Observacional - } \\
\text { Quantitativo }\end{array}$ & $\begin{array}{l}\text { Estudo analítico, } \\
\text { transversal }\end{array}$ & Zdravniški Vestnik/0,145 & Eslovênia & Inglesa & $\begin{array}{l}\text { Foi avaliada a presença da DMRA em } 45 \\
\text { gestantes até o } 3^{\circ} \text { dia pós-parto, e } \\
\text { comparadas com } 15 \text { mulheres não } \\
\text { grávidas. }\end{array}$ & $\begin{array}{l}\text { Incidência da DMRA em } \\
\text { mulheres gestantes e no pós-parto } \\
\text { imediato. }\end{array}$ \\
\hline $\begin{array}{l}\text { 8. Maryam } \\
\text { Saligheh et al. }\end{array}$ & 2017 & $\begin{array}{c}\text { Descritiva documental - } \\
\text { Quantitativo }\end{array}$ & Revisão sistemática & $\begin{array}{l}\text { Archives of Women's } \\
\text { Mental Health/1,111 }\end{array}$ & Austrália & Inglesa & Sem intervenção & $\begin{array}{c}\text { Exercício ou atividade física } \\
\text { podem ajudar a melhorar a } \\
\text { depressão pós-parto e a perda de } \\
\text { peso. } \\
\end{array}$ \\
\hline $\begin{array}{c}\text { 9. Hedwig Neels } \\
\text { et al. }\end{array}$ & 2018 & $\begin{array}{c}\text { Observacional - } \\
\text { Quantitativo }\end{array}$ & $\begin{array}{c}\text { Estudo } \\
\text { Observacional } \\
\text { Prospectivo }\end{array}$ & $\begin{array}{c}\text { European Journal of } \\
\text { Obstetrics, Gynecology } \\
\text { and Reproductive } \\
\text { Biology/ } / 0,84 \\
\end{array}$ & Bélgica & Inglesa & Sem intervenção & $\begin{array}{c}\text { Erros comuns cometidos na } \\
\text { tentativa de contrair os músculos } \\
\text { do assoalho pélvico em mulheres } \\
\text { logo após o parto. } \\
\end{array}$ \\
\hline $\begin{array}{l}\text { 10. Ana Carolina } \\
\text { Sartorato } \\
\text { Beleza et al. }\end{array}$ & 2017 & $\begin{array}{c}\text { Intervenção Clínica - } \\
\text { Quantitativo }\end{array}$ & $\begin{array}{l}\text { Ensaio Clínico } \\
\text { Randomizado e } \\
\text { Controlado }\end{array}$ & Physiotherapy/1,085 & Brasil & Inglesa & $\begin{array}{l}\text { Puérperas que relataram dor no período } \\
\text { pós-parto após o parto vaginal com } \\
\text { episiotomia. O grupo experimental }(\mathrm{n}=24) \\
\text { aplicou uma bolsa de gelo na região } \\
\text { perineal por } 20 \text { minutos. O grupo controle }\end{array}$ & $\begin{array}{c}\text { Efeito da crioterapia no alívio da } \\
\text { dor perineal após parto vaginal } \\
\text { com episiotomia. }\end{array}$ \\
\hline
\end{tabular}


Research, Society and Development, v. 10, n. 3, e47310312849, 2021

(CC BY 4.0) | ISSN 2525-3409 | DOI: http://dx.doi.org/10.33448/rsd-v10i3.12849

\begin{tabular}{|c|c|c|c|c|c|c|c|c|}
\hline & & & & & & & $\begin{array}{l}(\mathrm{n}=26) \text { recebeu atendimento rotineiro da } \\
\text { maternidade. Ambos os grupos foram } \\
\text { avaliados antes, após a remoção do saco } \\
\text { de gelo, e uma hora após o tratamento } \\
\text { com crioterapia. }\end{array}$ & \\
\hline $\begin{array}{l}\text { 11. Farzaneh } \\
\text { Ashrafinia et } \\
\text { al. }\end{array}$ & 2015 & $\begin{array}{l}\text { Intervenção Clínica - } \\
\text { Quantitativo }\end{array}$ & $\begin{array}{l}\text { Ensaio Clínico } \\
\text { Randomizado e } \\
\text { Controlado }\end{array}$ & $\begin{array}{l}\text { Singapore Medical } \\
\text { Journal/0,452 }\end{array}$ & Irã & Inglesa & $\begin{array}{l}\text { Puérperas } 72 \text { horas após parto vaginal. } \\
\text { Grupo intervenção }(\mathrm{n}=40) \text { e o grupo } \\
\text { controle }(\mathrm{n}=40 \text { ). No grupo de intervenção, } \\
\text { as mulheres realizaram exercícios de } \\
\text { Pilates cinco vezes por semana ( } 30 \text { min } \\
\text { por sessão) durante oito semanas } \\
\text { consecutivas. O grupo controle recebeu } \\
\text { sessão de treinamento sobre cuidados pós- } \\
\text { parto. } \\
\text {. }\end{array}$ & $\begin{array}{l}\text { Efeito dos exercícios de Pilates na } \\
\text { fadiga materna no pós-parto. }\end{array}$ \\
\hline $\begin{array}{l}\text { 12. Dayana Maia } \\
\text { Saboia et al. }\end{array}$ & 2018 & $\begin{array}{l}\text { Descritiva documental- } \\
\text { Quantitativo }\end{array}$ & Revisão sistemática & $\begin{array}{l}\text { Revista Brasileira de } \\
\text { Enfermagem } / 0,235\end{array}$ & Brasil & Portuguesa & Sem intervenção & $\begin{array}{l}\text { Eficácia das intervenções } \\
\text { realizadas no pós-parto para } \\
\text { prevenir incontinência urinária. }\end{array}$ \\
\hline $\begin{array}{l}\text { 13. Dorota Torbé } \\
\text { et al. }\end{array}$ & 2016 & $\begin{array}{l}\text { Descritiva documental - } \\
\text { Qualitativo }\end{array}$ & Revisão narrativa & $\begin{array}{l}\text { Pomeranian Journal of } \\
\text { Life Sciences/0,106 }\end{array}$ & Polônia & Polonesa & Sem intervenção & $\begin{array}{l}\text { Princípios da realização de } \\
\text { atividade física no puerpério } \\
\text { imediato, com foco na prevenção } \\
\text { dos efeitos da imobilização, no } \\
\text { treinamento muscular dos } \\
\text { abdominais e transversais e na } \\
\text { correta ativação dos músculos do } \\
\text { assoalho pélvico. }\end{array}$ \\
\hline $\begin{array}{l}\text { 14. Sumian Yang } \\
\text { et al. }\end{array}$ & 2017 & $\begin{array}{l}\text { Intervenção Clínica - } \\
\text { Quantitativo }\end{array}$ & $\begin{array}{l}\text { Ensaio Clínico } \\
\text { Randomizado e } \\
\text { controlado }\end{array}$ & $\begin{array}{c}\text { Journal of } \\
\text { Clinical Nursing } / 0,768\end{array}$ & China & Inglesa & $\begin{array}{l}189 \text { primíparas divididas em grupo } \\
\text { controle (n=60) que receberam somente } \\
\text { orientação pós-parto de rotina } 2 \text { horas pós- } \\
\text { parto, por } 1 \text { hora, grupo de treinamento } \\
\text { (n=63) que realizaram exercícios de Kegel } \\
\text { e movimentos pélvicos, } 2 \text { dias após o } \\
\text { parto até } 3 \text { meses pós o parto e o grupo } \\
\text { combinação (n=66) que receberam } \\
\text { estimulação elétrica intravaginal de baixa } \\
\text { frequência e voltagem ( } 3 \text { vezes por } \\
\text { semana por } 30 \text { minutos) a partir da sexta } \\
\text { semana pós-parto, além de realizar } \\
\text { exercícios de Kegel. }\end{array}$ & $\begin{array}{c}\text { Recuperação da função do tecido } \\
\text { nervoso pélvico no pós-parto após } \\
\text { a realização de exercícios de } \\
\text { Kegel combinados com } \\
\text { estimulação elétrica intravaginal } \\
\text { de baixa frequência e voltagem, } \\
\text { em mulheres que apresentaram } \\
\text { lesão nos músculos do assoalho } \\
\text { pélvico. }\end{array}$ \\
\hline $\begin{array}{l}\text { 15. Farzaneh } \\
\text { Ashrafinia et } \\
\text { al. }\end{array}$ & 2013 & $\begin{array}{l}\text { Intervenção Clínica - } \\
\text { Quantitativo }\end{array}$ & $\begin{array}{l}\text { Ensaio Clínico } \\
\text { Randomizado e } \\
\text { controlado }\end{array}$ & $\begin{array}{c}\text { Journal of Bodywork and } \\
\text { Movement } \\
\text { Therapies/0,47 }\end{array}$ & Irã & Inglesa & $\begin{array}{c}80 \text { mulheres no pós-parto foram } \\
\text { aleatoriamente divididas em dois grupos, } \\
\text { intervenção (GI=40) e controle }(\mathrm{GC}=40) \text {. } \\
\text { O GI realizou exercícios de Pilates com } \\
30 \text { min de duração, iniciados } 72 \text { horas } \\
\text { após o parto, cinco vezes por semana } \\
\text { durante } 8 \text { semanas consecutivas em } \\
\text { ambiente domiciliar. }\end{array}$ & $\begin{array}{c}\text { Efeito dos exercícios de Pilates na } \\
\text { melhora da qualidade do sono em } \\
\text { primigestas no pós-parto }\end{array}$ \\
\hline $\begin{array}{l}\text { 16. Seda Kayman- } \\
\text { Kose et al. }\end{array}$ & 2014 & $\begin{array}{l}\text { Intervenção Clínica - } \\
\text { Quantitativo }\end{array}$ & $\begin{array}{l}\text { Ensaio Clínico } \\
\text { Randomizado e } \\
\text { controlado }\end{array}$ & $\begin{array}{l}\text { The Journal of Maternal- } \\
\text { Fetal \& Neonatal } \\
\text { Medicine/ } / 0,671\end{array}$ & Turquia & Inglesa & $\begin{array}{l}100 \text { mulheres submetidas à cesariana } \\
\text { foram aleatoriamente designadas para o } \\
\text { grupo placebo (Grupo } 1 \text { ) ou o grupo }\end{array}$ & $\begin{array}{c}\text { Aplicação da estimulação elétrica } \\
\text { nervosa transcutânea (TENS) no } \\
\text { manejo da dor relacionada às }\end{array}$ \\
\hline
\end{tabular}


Research, Society and Development, v. 10, n. 3, e47310312849, 2021

(CC BY 4.0) | ISSN 2525-3409 | DOI: http://dx.doi.org/10.33448/rsd-v10i3.12849

\begin{tabular}{|c|c|c|c|c|c|c|c|c|}
\hline & & & & & & & $\begin{array}{l}\text { TENS (Grupo 2), enquanto } 100 \text { mulheres } \\
\text { que tiveram parto por via vaginal sem } \\
\text { episiotomia foram randomizadas para o } \\
\text { grupo placebo (Grupo } 3 \text { ) ou o grupo } \\
\text { TENS (Grupo 4). O TENS foi realizado } \\
\text { uma vez por } 30 \text { min após o parto ter sido } \\
\text { completado. Nenhuma corrente elétrica } \\
\text { foi transmitida aos grupos placebo, nos } \\
\text { grupos de tratamento o TENS foi } \\
\text { gradualmente aplicado na frequência de } \\
100 \mathrm{~Hz} \text { a partir de } 0 \text { mA e estabilizada no } \\
\text { valor para o qual o paciente sentiu alívio, } \\
\text { sem qualquer desconforto. } \\
\end{array}$ & $\begin{array}{c}\text { contrações uterinas após o parto } \\
\text { vaginal e cesáreo }\end{array}$ \\
\hline $\begin{array}{l}\text { 17. Ligia de Sousa } \\
\text { et al. }\end{array}$ & 2014 & $\begin{array}{c}\text { Intervenção Clínica - } \\
\text { Quantitativo }\end{array}$ & $\begin{array}{c}\text { Ensaio Clínico } \\
\text { Randomizado e } \\
\text { controlado }\end{array}$ & $\begin{array}{c}\text { Journal of Obstetrics } \\
\text { and Gynaecology/ } \\
0,305\end{array}$ & Brasil & Inglesa & $\begin{array}{l}32 \text { mulheres no pós-parto que estavam } \\
\text { amamentando foram randomizadas em } \\
\text { grupo experimental (GE=16) e controle } \\
\text { (GC=16). Na primeira avaliação, as } \\
\text { mulheres no pós-parto do GE e GC foram } \\
\text { monitoradas durante a primeira } \\
\text { amamentação e questionadas quanto ao } \\
\text { grau de dor da contração uterina. Na } \\
\text { segunda avaliação, o GE usou TENS. Os } \\
\text { eletrodos foram colocados entre T10-L1 e } \\
\text { S2-S4, com frequência de } 100 \mathrm{~Hz}, \\
\text { duração de pulso de } 75 \mu \text { s e intensidade } \\
\text { ajustada para produzir uma sensação forte } \\
\text { e tolerável por } 40 \text { min. }\end{array}$ & $\begin{array}{c}\text { Uso da estimulação elétrica } \\
\text { nervosa transcutânea (TENS) no } \\
\text { alívio da dor ocasionada pela } \\
\text { contração uterina durante a } \\
\text { amamentação em mulheres } \\
\text { multíparas. }\end{array}$ \\
\hline
\end{tabular}

Fonte: Elaborado pelas autoras. 


\section{Discussão}

Dentre as principais intervenções realizadas no puerpério imediato houve um predomínio da utilização dos exercícios para diminuição da DMRA, com 5 artigos que abordavam a ativação da musculatura do transverso abdominal, e destes, apenas 1 estudo associava a contração dos MAPS. Ao se avaliar de forma longitudinal a incidência da DMRA em gestantes até o $3^{\circ}$ dia pós-parto, Medjimurec, Mihelj, Šćepanović, (2018) identificaram uma prevalência de 38,1\%, sendo a maioria delas localizadas acima da cicatriz umbilical.

Na revisão de literatura realizada por Mota et al. (2015), cujo objetivo era revisar criticamente os fatores de risco, as implicações funcionais e o efeito do exercício na DMRA durante a gestação e após o parto, identificou-se que não há fatores de riscos significativos para o surgimento da DMRA, bem como as mulheres que apresentavam esta alteração não estavam mais propensas a dor lombopélvica, e apontaram na literatura uma lacuna relacionada aos mecanismos de diferentes exercícios abdominais para prevenção e tratamento da DMRA. Já na revisão sistemática de Warner e Dayan (2018), os estudos encontrados apontavam um melhor potencial para restauração da forma e da função da parede abdominal quando os exercícios tinham enfoque no músculo transverso abdominal, sendo que os resultados variaram de acordo com fatores individuais, propriocepção, controle motor e co-contração dos MAP.

Ainda sobre as intervenções encontradas na literatura para tratamento da DMRA, foi encontrado outro artigo de revisão que tinha como objetivo propor procedimentos fisioterapêuticos baseados na literatura disponível, que traz como melhor opção de tratamento a realização de um protocolo fisioterapêutico que obteve melhor êxito na literatura, o qual consiste na realização de uma flexão anterior de tronco, em decúbito dorsal, associado a uma expiração lenta e prolongada, e ao iniciar a inspiração, lentamente se retorna a posição inicial, sendo recomendada a realização deste exercício duas vezes por dia, em até 10 repetições durante a gestação, e depois do terceiro dia pós-parto, realizar o mesmo exercício 5 vezes por dia, cerca de 10 repetições, porém, os autores reforçam que existem poucos referências científicas acerca desse tratamento (Opala-Berdzik \& Medjimurec, 2009).

E por fim, o único estudo com essa temática que realizou um ensaio clínico controlado e randomizado, foi composto por 40 mulheres, com até 3 dias pós-parto, divididas igualmente em dois grupos, alocadas aleatoriamente em um grupo controle e intervenção, que realizou durante 6 semanas um protocolo fisioterapêutico baseado na realização de uma série de exercícios divididos em três partes: $1^{\circ}$ - Manter uma postura sustentada por 20 minutos, $2^{\circ}$ - Realizar a flexão anterior do tronco, depois completar essa flexão curvando a cabeça em direção a coxa, e em seguida flexionar e estender os joelhos, associando cada movimento a uma expiração lenta e profunda, cada exercício foi repetido 10 vezes, com duração de 10 segundos/cada em decúbito dorsal, com pernas e joelhos flexionados, mantendo pés apoiados na maca, $3^{\circ}$ - Programa educativo focado na prevenção. Como conclusão deste estudo, os autores apontam que esse novo programa fisioterapêutico é um método eficaz no tratamento da DMRA em mulheres pós-parto (Bobowik \& Dąbek, 2018).

A TENS foi outra modalidade muito utilizada no puerpério imediato, no estudo realizado por Kayman-Kose et al. (2014), eles avaliaram o manejo da dor relacionada às contrações uterinas após o parto vaginal, às contrações uterinas e a dor relacionada a incisão abdominal após cesariana. A TENS foi realizada uma vez por 30 min após o parto ter sido completado, com frequência (F) de $100 \mathrm{~Hz}$, o nível de dor foi medido pela escala visual analógica (EVA) e escala numérica verbal (ENV). Foi observado que o grupo que recebeu TENS apresentou escores na EVA e na ENV estatisticamente inferiores ao grupo placebo.

Outro estudo realizado por Alves et al. (2015), mensurou apenas o nível da dor na incisão da cesariana antes e após a aplicação do TENS, com os seguintes parâmetros F: $100 \mathrm{~Hz}$ e largura de pulso 100 s, durante 30 minutos e classificaram o 
nível de dor através da EVA. Ao comparar a intensidade da dor antes e após a TENS, foi encontrada uma diminuição significativa nos valores, deixando claro sua eficácia na redução do quadro álgico de dores agudas no pós-operatório de cesariana.

A TENS também foi utilizada de forma eficaz para aliviar a dor da contração uterina durante a amamentação, os eletrodos foram colocados entre T10-L1 e S2-S4, com frequência $100 \mathrm{~Hz}$, largura de pulso de $75 \mu$ s e intensidade ajustada para produzir uma sensação forte e tolerável por 40 min (Sousa, Gomes-Sponholz \& Nakano, 2014).

Referente ao treinamento da musculatura do assoalho pélvico, Neels, De Wachter, Wyndaele, Van Aggelpoel, Vermandel, (2018) objetivaram observar a prevalência de contração de outros músculos e realização de outros movimentos, que foram denominados os erros mais comuns na tentativa de contrair os MAPS, durante os primeiros dias após o parto. O estudo incluiu 382 mulheres no primeiro ao sexto dia pós-parto, que foram orientadas a realizar contração desta musculatura sendo avaliadas com observação visual. Duzentos e dezesseis puérperas (57\%) mostraram contração de outros músculos e realização de outros movimentos, concluindo assim, que os erros podem reduzir a capacidade de contrair os MAPS, mas podem facilmente ser desaprendidos com observação visual e feedback verbal.

Nos estudos que pesquisaram acerca da prevenção e tratamento da IU (incontinência urinária), a revisão sistemática de Mørkved e Bø (2014) inferiu que o treinamento dos músculos do assoalho pélvico durante a gravidez e após o parto pode prevenir e tratar a incontinência urinária. Um protocolo de treinamento seguindo princípios gerais de treinamento de força, enfatizando contrações máximas e pelo menos em um período de treinamento de 8 semanas pode ser recomendado. Corroborando com este estudo, a revisão sistemática de Saboia et al. (2018) concluiu que há evidências de que programas de treinamento dos MAPS realizados tanto no pós-parto imediato quanto no pós-parto tardio resultam em aumento significativo da força muscular e contribuem para a prevenção da incontinência urinária. Programas de exercícios que utilizam mais séries de repetições, com mais contrações e maior tempo de sustentação da contração parecem ser os mais indicados.

Em relação ao Pilates, foi evidenciado que melhorou a qualidade do sono e o nível de fadiga nas puérperas, as participantes do estudo começaram a fazer os exercícios a partir de 72 horas do pós-parto e continuaram por 8 semanas. Os exercícios foram realizados no início da manhã por 30 min após a amamentação, 5 dias por semana e observou-se que após 8 semanas, o grupo de intervenção teve efeitos benéficos na melhora da qualidade do sono e redução dos níveis de fadiga no pósparto (Ashrafinia, et al., 2013).

Concernente ao exercício físico, a maioria estudos relataram mudanças em apenas um desfecho, a depressão pósparto. As pesquisas implementaram diferentes modalidades de exercício, sendo a mais abordada a caminhada e as mais propensas a associar depressão com perda de peso foram as que utilizaram exercícios com supervisão, frequência semanal, duração programada (30 a 150 minutos por semana) e intensidade moderada. Toda via, Saligheh, Hackett, Boyce, Cobley, (2017), evidenciaram que houve inconsistência na redução dos sintomas de depressão e da perda de peso.

Já uma revisão narrativa descreveu os princípios da prática de atividade física no período do puerpério precoce, afirmando que a atividade física no puerpério imediato quando indicada e dosada adequadamente, auxilia na regressão mais rápida das mudanças fisiológicas associadas ao período da gravidez e do parto. Exercícios leves e delicados devem ser iniciados o mais cedo possível, ainda na posição horizontal, uma vez que nos primeiros dias após o parto, as alterações puerperais são mais intensas e estes facilitam e aceleram o ritmo de retorno a condições pré-gravídicas de forma significativa (Torbé, Stolarek, Lubkowska \& Torbé, 2016).

Outro recurso empregado no puerpério imediato foi a crioterapia, um ensaio clínico controlado randomizado investigou o uso da crioterapia no alívio da dor perineal em mulheres após parto vaginal com episiotomia. O estudo incluiu 50 mulheres que relataram dor no período pós-parto após o parto vaginal com episiotomia, as puérperas tinham no mínimo 6 
Research, Society and Development, v. 10, n. 3, e47310312849, 2021

(CC BY 4.0) | ISSN 2525-3409 | DOI: http://dx.doi.org/10.33448/rsd-v10i3.12849

horas e no máximo 24 horas após o parto. As mulheres do grupo experimental aplicaram uma bolsa de gelo picado na região perineal por 20 minutos, enquanto que o grupo controle recebeu atendimento rotineiro da maternidade. A queixa de dor foi avaliada por meio de uma escala numérica de avaliação da dor (0 a 10), a temperatura perineal foi medida usando um termômetro infravermelho, e a satisfação das mulheres submetidas ao tratamento foi avaliada por meio de um questionário. O estudo concluiu que após 20 minutos de aplicação, a crioterapia foi eficaz no alívio da dor perineal em mulheres no pós-parto imediato após o parto vaginal com episiotomia e a maioria das mulheres que receberam a intervenção declarou que a usariam novamente (Beleza, Ferreira, Driusso, Dos Santos \& Nakano, 2017).

Com intuito de diminuir possíveis disfunções acometidas durante o parto, como prolapso de órgãos pélvicos (POP) e IU, os EK foram utilizados para recuperação da função do tecido nervoso pélvico pós-parto tanto de forma isolada, como combinados com DES. Os EK foram iniciados 2 dias após o parto, de 2 a 3 vezes por dia e a DES foi realizado 15 vezes (3 vezes por semana por 30 minutos, frequência $60-80 \mathrm{~Hz}$ e largura de pulso de 20-40 $\mu$ s) a partir da sexta semana pós-parto. Este estudo demostrou que esses exercícios combinados aumentaram a força e o potencial muscular do assoalho pélvico, reduzindo o aparecimento de POP e IU (Yang, et al., 2017).

\section{Conclusão}

Foi evidenciado diante dessa pesquisa bibliométrica que as intervenções fisioterapêuticas realizadas no puerpério imediato nos últimos 10 anos abordaram, em sua maioria, a temática de avaliação e tratamento da diástase dos músculos retoabdominais, seguido pelo uso da eletroestimulação transcutânea para alívio da dor no pós-parto imediato e treinamento da musculatura do assoalho pélvico. Além disso, ficou claro que a produção científica na temática abordada vem aumentando nos últimos cinco anos, principalmente no Brasil, onde foi produzido o maior número de estudos.

Em contrapartida, os resultados encontrados apontam para diversas lacunas, no que tange a intervenção fisioterapêutica no puerpério imediato, tendo em vista que outras intervenções poderiam ter sido realizadas, como as orientações posturais para as atividades de vida diária e amamentação, cuidados com as mamas, massagens relaxantes, e diversas outras abordagens que podem ser realizadas na prática clínica, e que necessitam de mais estudos que abordem protocolos bem definidos e metodologias bem estabelecidas, visando o tratamento fisioterapêutico voltado apenas para mulheres no puerpério imediato.

\section{Referências}

Alves EMS, Rabêlo TN, Santos MGR, Souza IG, Lima PAL, Santana LS. (2015). Transcutaneous electric nerve stimulation for post-Cesarean section analgesia. Revista Dor, 16(4): 263-6. https://doi.org/10.5935/1806-0013.20150053.

Andrade RD, Santos JS, Maia MAC, Mello DF. (2015). Fatores relacionados à saúde da mulher no puerpério e repercussões na saúde da criança. Escola Anna Nery Revista de Enfermagem, 19(1), 181-6. https://doi.org/10.5935/1414-8145.20150025.

Araújo CA. (2006). Bibliometria: evolução histórica e questões atuais. Em questão, 12(1): 11-32. https://seer.ufrgs.br/EmQuestao/article/view/16/5.

Ashrafinia F, Mirmohammadali M, Rajabi H, Kazemnejad A, Haghighi KS, Amelvalizadeh M. (2015). Effect of Pilates exercises on postpartum maternal fatigue. Singapore Medical Journal, 56(3), 169-73. DOI: 10.11622/smedj.2015042.

Ashrafinia F, Mirmohammadali M, Rajabi H, Kazemnejad A, Sadeghniiathaghighi K, Amelvalizadeh M, Chen, H. (2013). The effects of Pilates exercise on sleep quality in postpartum women. Journal of Bodywork and Movement Therapies, 18(2): 190-9. https://doi.org/10.1016/j.jbmt.2013.09.007.

Beleza ACS, Ferreira CHJ, Driusso P, Dos Santos CB, Nakano AMS. (2017). Effect of cryotherapy on relief of perineal pain after vaginal childbirth with episiotomy: a randomized and controlled clinical trial. Physiotherapy, 103(4): 453-8. https://doi.org/10.1016/j.physio.2016.03.003.

Bobowik PŻ, Dąbek A. (2018). Physiotherapy in women with diastasis of the rectus abdominis muscles. Advances in Rehabilitation, (3): 11-7. https://doi.org/10.5114/areh.2018.80964. 
Research, Society and Development, v. 10, n. 3, e47310312849, 2021

(CC BY 4.0) | ISSN 2525-3409 | DOI: http://dx.doi.org/10.33448/rsd-v10i3.12849

Brasil. (2011). Política Nacional de Atenção Integral à Saúde da Mulher: Princípios e diretrizes. Brasília: Ministério da saúde, Secretaria de Atenção à Saúde. https://bvsms.saude.gov.br/bvs/publicacoes/politica_nacional_mulher_principios_diretrizes.pdf.

Burti JS, Cruz JPS, Silva AC, Moreira IL. (2016). Assistência ao puerpério imediato: o papel da fisioterapia. Revista da Faculdade de Ciências Médicas de Sorocaba, 18(4): 193-8. https://doi.org/10.5327/Z1984-4840201625440.

Crockett KL, Bowen A, Madill SJ, Kumaran M, Epp C, Graham AM. (2018). A review of the effects of physical therapy on self-esteem in postpartum women with lumbopelvic dysfunction. Journal of Obstetrics and Gynaecology Canada, 40(11), 1-12. https://www.jogc.com/article/S1701-2163(18)30589-9/fulltext.

Evenson KR, Mottola MF, Owe KM, Rousham EK, Brown WJ. (2014). Summary of international guidelines for physical activity following pregnancy. Obstetrical \& Gynecological Survey, 69(7), 407-14. DOI: 10.1097/OGX.00000000000000077.

Falcão KPM, Carvalho ACF de, Marques ACML, Vieira AG, Barros JD, Rolim RB. (2015). Prevalência de alterações posturais em puérperas frente ao posicionamento durante a amamentação. Revista de Enfermagem UFPE on line, 9(11), 9839-45. https://doi.org/10.5205/1981-8963-v9i11a10776p9839-98452015.

Gardenghi LA, Dezotti NR, Dalio MB, Joviliano EE, Piccinato CE. (2017). Gestational lower limb edema and venous reflux in healthy primigravidae. International Angiology, 36(6), 569-73. DOI: 10.23736/S0392-9590.17.03865-2.

Goyal RC, Banginwar AS, Ziyo F, Toweir AA. (2011). Breastfeeding practices: positioning, attachment (latch-on) and effective suckling - A hospital-based study in Libya. Journal of Family \& Community Medicine, 18(2), 74-9. https://www.jfcmonline.com/article.asp?issn=22308229; year $=2011$; volume $=18 ;$ issue $=2 ;$ spage $=74$; epage $=79$; aulast $=$ Goyal .

Haran C, Van Driel M, Mitchell BL, Brodribb WE. (2014). Clinical guidelines for postpartum women and infants in primary care-a systematic review. BMC Pregnancy and Childbirth, 14(1), 1-9. https://bmcpregnancychildbirth.biomedcentral.com/articles/10.1186/1471-2393-14-51.

Kayman-Kose S, Arioz DT, Toktas H, Koken G, Kanat-Pektas M, Kose M, Yilmazer M. (2014). Transcutaneous electrical nerve stimulation (TENS) for pain control after vaginal delivery and cesarean section. The Journal of Maternal-Fetal \& Neonatal Medicine, 27(15): 1572-5. https://doi.org/10.3109/14767058.2013.870549.

Lavand'homme P. (2013). Chronic pain after childbirth. Current Opinion in Anesthesiology, 26(3): 273-7. https://journals.lww.com/coanesthesiology/Abstract/2013/06000/Chronic_pain_after_childbirth.5.aspx.

Lemos A, Souza AI, Andrade AD, Figueiroa JN, Filho JEC. (2011). Pregnancy inter-recti abdominis distance has no impact on respiratory strength. Journal of Physical Therapy Science, 23(5), 757-60. https://doi.org/10.1589/jpts.23.757.

Machado RN. (2007). Análise cientométrica dos estudos bibliométricos publicados em periódicos da área de biblioteconomia e ciência da informação (19902005). Perspectivas em Ciência da Informação, 12(3); 2-20. https://doi.org/10.1590/S1413-99362007000300002.

Martinez-Pulgarin DF, Acevedo-Mendoza WF, Cardona-Ospina JA, Rodríguez-Morales AJ, Paniz-Mondolfi AE. (2016). A bibliometric analysis of global Zika research. Travel Medicine and Infectious Disease, 14(1): 55-7. https://www.sciencedirect.com/science/article/pii/S1477893915001209?via\%3Dihub.

Medjimurec MS, Mihelj E, Šćepanović D. (2018). The incidence and location of diastasis rectus abdominis during the childbearing year and puerperium at the Maternity Hospital Ljubljana. Zdravniški Vestnik, 87(6): 215-22. https://doi.org/10.6016/ZdravVestn.2450.

Mesquita NS, Rodrigues DP, Monte AS, Ferreira ALA, Manguinho CPC, Brandão JC. (2019). Percepções de puérperas acerca do cuidado de enfermagem recebido no pós-parto imediato. Revista de pesquisa: Cuidado é fundamental, 11(1), 160-6. http://www.seer.unirio.br/index.php/cuidadofundamental/article/view/6851/pdf.

Mørkved S, Bø K. (2014). Effect of pelvic floor muscle training during pregnancy and after childbirth on prevention and treatment of urinary incontinence: a systematic review. British Journal of Sports Medicine, 48(4): 299-310. http://dx.doi.org/10.1136/bjsports-2012-091758.

Mota P, Pascoal AG, Bo K. (2015). Diastasis recti abdominis in pregnancy and postpartum period. Risk factors, functional implications and resolution. Current Women's Health Reviews, 11(1): 59-67. DOI: 10.2174/157340481101150914201735.

Neels H, De Wachter S, Wyndaele JJ, Van Aggelpoel T, Vermandel A. (2018). Common errors made in attempt to contract the pelvic floor muscles in women early after delivery: a prospective observational study. European Journal of Obstetrics \& Gynecology and Reproductive Biology, 220(1): 113-7. https://doi.org/10.1016/j.ejogrb.2017.11.019.

Opala-Berdzik A, Dąbrowski S. (2009). Physiotherapy in diastasis of the rectus muscles of abdomen in women during pregnancy and postpartum. Physiotherapy, 17(4): 67-70. https://www.deepdyve.com/lp/de-gruyter/physiotherapy-in-diastasis-of-the-rectus-muscles-of-abdomen-in-womename8ReA7RL.

Pinto AVA, Schleder JC, Penteado C, Gallo RBS. (2015). Avaliação da mecânica respiratória em gestantes. Fisioterapia e Pesquisa, 22(4), 348-54. https://doi.org/10.590/1809-2950/13667922042015.

Pizzoferrato AC, Fauconnier A, Bader G, de Tayrac R, Fort J, Fritel X. (2016). Is prenatal urethral descent a risk factor for urinary incontinence during pregnancy and the postpartum period? International Urogynecology Journal, 27(7), 1003-11. https://link.springer.com/article/10.1007\%2Fs00192-015-2918-Z.

Rett MT, Almeida TV, Mendonça ACR, Santana JM, Ferreira APL, Araújo KCGM. (2014). Fatores materno-infantis associados à diástase dos músculos retos do abdome no puerpério imediato. Revista Brasileira de Saúde Materno Infantil, 14(1), 73-80. https://doi.org/10.1590/S1519-38292014000100007.

Rett MT, Bernardes NO, Santos AM, Oliveira MR, Andrade SC. (2008). Atendimento de puérperas pela fisioterapia em uma maternidade pública humanizada. Fisioterapia e Pesquisa, 15(4), 361-6. http://dx.doi.org/10.1590/S1809-29502008000400008. 
Research, Society and Development, v. 10, n. 3, e47310312849, 2021

(CC BY 4.0) | ISSN 2525-3409 | DOI: http://dx.doi.org/10.33448/rsd-v10i3.12849

Saboia DM, Bezerra KC, Neto JAV, Bezerra LRPS, Oriá MOB, Vasconcelos CTM. (2018). Eficácia das intervenções realizadas no pós-parto para prevenir incontinência urinária: revisão sistemática. Revista Brasileira de Enfermagem, 71(3): 1544-52. https://doi.org/10.1590/0034-7167-2017-0338.

Saleh MSM, Botla AMM, Elbehary NAM. (2019). Effect of core stability exercises on postpartum lumbopelvic pain: a randomized controlled trial. Journal of Back and Musculoskeletal Rehabilitation, 32(2), 205-13. DOI: 10.3233/BMR-181259.

Saligheh M, Hackett D, Boyce P, Cobley S. (2017). Can exercise or physical activity help improve postnatal depression and weight loss? A systematic review. Archives of Women's Mental Health, 20(5): 595-611. DOI: 10.1007/s00737-017-0750-9.

Santana LS, Gallo RBS, Marcolin AC, Ferreira CHJ, Quintana SM. (2011). Utilização dos recursos fisioterapêuticos no puerpério: revisão da literatura. Feminina, 39(5), 245-50. http://files.bvs.br/upload/S/0100-7254/2011/v39n5/a2506.pdf.

Siddiqui AH, Tauheed N, Ahmad A, Mohsin Z. (2014). Função pulmonar em mulheres com gestação única ou gemelar avançada e sem complicações. Jornal Brasileiro de Pneumologia, 40(3), 244-9. https://doi.org/10.1590/S1806-37132014000300007.

Sousa L, Gomes-Sponholz FA, Nakano AM. (2014). Transcutaneous electrical nerve stimulation for the relief of post-partum uterine contraction pain during breast-feeding: a randomized clinical trial. The Journal of Obstetrics and Gynaecology Research, 40(5): 1317-23. https://doi.org/10.1111/jog.12345.

Teymuri Z, Hosseinifar M, Sirousi M. (2018). The effect of stabilization exercises on pain, disability, and pelvic floor muscle function in postpartum lumbopelvic pain: a randomized controlled trial. American Journal of Physical Medicine \& Rehabilitation, 97(12), 885-91. https://journals.lww.com/ajpmr/Fulltext/2018/12000/The_Effect_of_Stabilization_Exercises_on_Pain,.5.aspx.

Theodorsen NM, Strand LI, B $\emptyset$ K. (2018). Effect of pelvic floor and transversus abdominis muscle contraction on inter-rectus distance in postpartum women: a cross-sectional experimental study. Physiotherapy, 105(3), 315-20. https://www.physiotherapyjournal.com/article/S0031-9406(18)30311-0/fulltext.

Torbé D, Stolarek A, Lubkowska A, Torbé A. (2016). Physical activity recommended in the early postpartum period. Pomeranian Journal of Life Sciences, 62(3):

https://www.researchgate.net/publication/317226858_Aktywnosc_fizyczna_zalecana_we_wczesnym_pologu/fulltext/59335fbe0f7e9beee7ad5aed/Aktywnoscfizyczna-zalecana-we-wczesnym-pologu.pdf.

Unsgaard-Tøndel M, Vasseljen O, Woodhouse A, Mørkved S. (2016). Exercises for women with persistent pelvic and low back pain after pregnancy. Global Journal of Health Science, 8(9), 107-20. DOI: 10.5539/gjhs.v8n9p107.

Vanderkruik RC, Tunçalp Ö, Chou D, Say L. (2013). Framing maternal morbidity: WHO scoping exercise. BMC Pregnancy and Childbirth, 13(1), 1-7. https://bmcpregnancychildbirth.biomedcentral.com/articles/10.1186/1471-2393-13-213.

Werner LA, Dayan M. (2019). Diastasis recti abdominis-diagnosis, risk factors, effect on musculoskeletal function, framework for treatment and implications for the pelvic floor. Current Women's Health Reviews, 15(2): 86-101. DOI: 10.2174/1573404814666180222152952.

Witt AM, Bolman M, Kredit S, Vanic A. (2016). Therapeutic breast massage in lactation for the management of engorgement, plugged ducts, and mastitis. Journal of Human Lactation, 32(1), 123-31. https://journals.sagepub.com/doi/10.1177/0890334415619439?url_ver=Z39.88$2003 \&$ rfr_id=ori:rid:crossref.org\&rfr_dat=cr_pub\%20\%200pubmed.

Wu YM, McInnes N, Leong Y. (2018). Pelvic floor muscle training versus watchful waiting and pelvic floor disorders in postpartum women: A systematic review and meta-analysis. Female Pelvic Medicine \& Reconstructive Surgery, 24(2), 142-9. DOI: 10.1097/SPV.0000000000000513.

Yang S, Sang W, Feng J, Zhao H, Li X, Li P, Fan H, Tang Z, Gao L. (2017). The effect of rehabilitation exercises combined with direct vagina low voltage low frequency electric stimulation on pelvic nerve electrophysiology and tissue function in primiparous women: a randomised controlled trial. Journal of Clinical Nursing, 26(24): 4537-47. https://doi.org/10.1111/jocn.13790. 